\title{
MMW-HOTLINE
}

Leser der MMW können sich mit allen Fragen zur Abrechnung und Praxisführung an Helmut Walbert, Facharzt für Allgemeinmedizin, Würzburg, wenden. Sie erreichen ihn jeden Donnerstag von 13 bis $15 \mathrm{Uhr}$ unter der kostenlosen Rufnummer (0800) 2379830 oder per E-Mail: w@lbert.info.

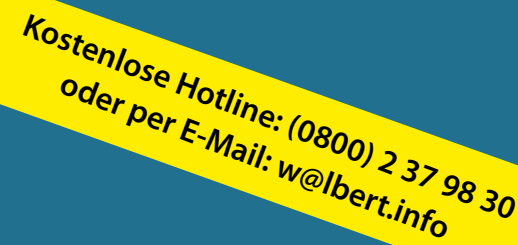

MFA nach Unfall nicht mehr voll einsetzbar

\section{Kann ich ihr kündigen?}

Dr. med. H. W., Arzt für Allgemeinmedizin, Berlin:

Eine Medizinische Fachangestellte (MFA) hatte einen schweren Verkehrsunfall und ist jetzt gehbehindert. Sie ist nach dem Schwerbehindertengesetz mit 30\% eingestuft. Vor dem Unfall war sie mit Arbeiten im Labor und der Assistenz beschäftig. Sie wäre eigentlich nur noch für die Anmeldung einsetzbar. Dort habe ich aber seit Jahren eine hervorragend auf diesen Arbeitsplatz spezialisierte Arbeitskraft, auf die ich nicht verzichten möchte. Wie gehe ich mit dieser Situation um? Kann ich der MFA kündigen?

Antwort: Eine nicht ganz einfache Situation! Nach einer relativ jungen Entscheidung des Landesarbeitsgerichts in Köln (AZ:: 2SWa 1276/10) hat ein Arbeitnehmer, der durch ein Ereignis plötzlich körperlich einge-

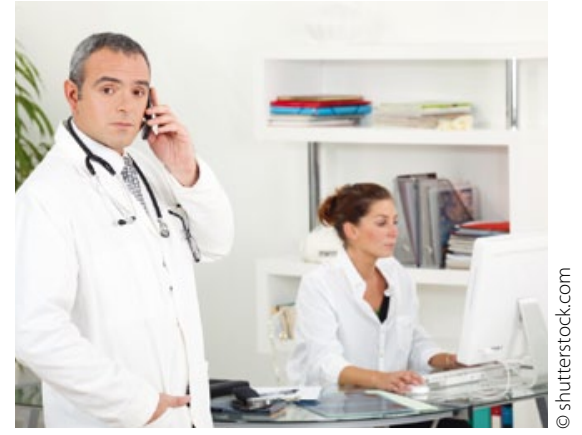

Muss die Praxisperle ihren Platz für eine Behinderte räumen?

schränkt ist, Anspruch auf Versetzung innerhalb des Betriebes. Es muss diesem Arbeitnehmer ein seiner Behinderung entsprechender Arbeitsplatz angeboten werden. Der Arbeitgeber ist nach diesem Urteil auf jeden Fall zu einer entsprechenden Prüfung verpflichtet, ob er dem Arbeitnehmer einen anderen, seiner Behinderung gerechten Arbeitsplatz anbieten kann. Kommen Sie dieser Verpflichtung als Arbeitgeber nicht nach, kann der Arbeitnehmer sogar Schadensersatz verlangen, so das Landesarbeitsgericht Köln.

Der Nachweis liegt beim Arbeitgeber. Sollte die MFA für den offensichtlich einzig verbleibenden Arbeitsplatz der Rezeption nicht geeignet sein oder die Fachkraft an der Anmeldung für andere Arbeiten in der Praxis nicht geeignet sein, rate ich vor einer Kündigung dringend zu einer Beratung durch einen entsprechenden Fachanwalt für Arbeitsrecht. Ich empfehle, die Kosten für den Anwalt nicht zu scheuen. Der Ärger in der Praxis - erst durch die fehlende verunfallte MFA und jetzt durch ihre eingeschränkte Einsatzfähigkeit - wird sonst noch unnötig teuer, wenn nicht jeder Schritt vorher durchdacht und abgesichert ist.

\section{Jetzt verlangt der Apotheker ein BtM-Rezept}

Frau Dr. med. G .P., Allgemeinärztin. KV Thüringen:

Ein Apotheker hat bei einer Patientin, die seit Jahren Tilidin-Tropfen auf normalem Rezept bekommen hat, auf ein Betäubungsmittelrezept bestanden. Warum muss Tilidin nun auf BtM-Rezepten verordnet werden?
Antwort: Nicht Tilidinan sich, sondern nur die flüssige Form muss auf ein BtM-Rezept. Der Missbrauch und die Abhängigkeit von flüssigen Zubereitungen der fixen Kombination aus Tilidin und Naloxon haben den Gesetzgeber dazu veranlasst, die flüssige Form ab Januar 2013 dem Betäubungsmittelrecht zu unterstellen.

Die Verordnung von flüssigem Tilidin nur noch als Betäubungsmittel (BtM) auf spe- ziellen BtM-Rezepten ist eine Entscheidung des Sachverständigenausschusses für BtM. Dieser hat die Empfehlung ausgesprochen, der die Bundesregierung unverzüglich zustimmte.

Nicht betroffen sind die festen Darreichungsformen von Tilidin/Naloxon mit verzögerter Wirkstofffreigabe, so genannte Retardpräparate. 